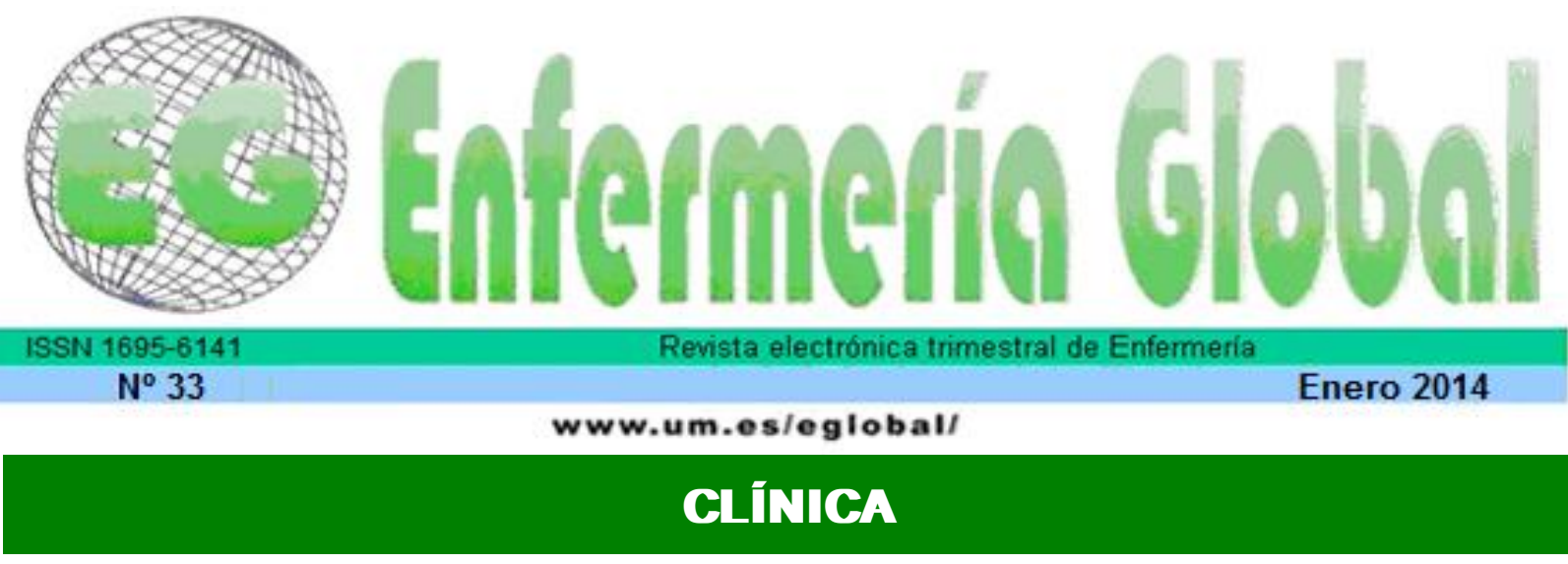

\title{
Condiciones de salud y funcionalidad de los ancianos del Valle Paraíba, São Paulo, Brasil
}

Condições de saúde e funcionalidade dos idosos do Vale Paraíba, São Paulo, Brasil

Health conditions and functionality of the elderly Valley Paraíba, Sao Paulo, Brazil

\section{*De Oliveira Araújo, Claudia Lysia **Mancussi e Faro, Ana Cristina}

\author{
*Doctora en Enfermería. Profesora Titular, Facultades Integradas Teresa de Ávila - Lorena, São Paulo \\ E-mail: claudialysia@usp.br ***Profesora Libre Docente del Departamento de Enfermería Médico- \\ Quirúrgica de la Escuela de Enfermería de la Universidad de São Paulo. Orientadora. Grupo de \\ Investigación, Rehabilitación, Funcionalidad y Educación en Salud. Brasil.
}

Palabras clave: Clasificación Internacional de Funcionalidad, Discapacidad y Salud; ancianos; centros
sociales y de ocio; envejecimiento; Enfermería; Gerontología.
Palavras chave: Classificação Internacional de Funcionalidade, Incapacidade e Saúde; Idoso; Centros
de Convivência e Lazer; Envelhecimento; Enfermagem; Gerontologia.
Keywords: International Classification of Functioning; Disability and Health; Aging; Social Centers and Leisure; Aging; Nursing; Gerontology..

\section{RESUMEN}

Los principios del envejecimiento con calidad de vida describen al anciano como proactivo, definiendo sus objetivos y esforzándose por alcanzarlos, reuniendo recursos que son útiles en la adaptación a los cambios y participar activamente en el mantenimiento de la salud.

El objetivo de esta investigación es caracterizar el perfil de las personas mayores que participan en el Centro Comunitario de Personas Mayores con respecto a sexo, edad, estado civil, origen étnico declarado, educación, ocupación, ingresos personales, número de hijos, nacionalidad, condiciones de vida, medios de transporte que utilizan para ir a la Corte Penal Internacional y actividad física preferida y describir las condiciones de salud y el rendimiento en actividades de la vida diaria de los participantes de mayor edad del Centro Comunitario de Personas Mayores, de acuerdo con la Clasificación Internacional del Funcionamiento, de la Discapacidad y la Salud (CIF).

Método: La investigación fue de tipo exploratorio, descriptivo, transversal, con enfoque cuantitativo, llevado a cabo en los Centros Comunitarios de Personas Mayores Paraíba Valley, São Paulo, Brasil. El estudio incluyó a aquellos que cumplieron con los criterios de inclusión de edad o mayores de 60 años, participando durante al menos tres meses y un máximo de un año de los Centros Comunitarios de Personas Mayores, que eran el campo de búsqueda. El estudio se caracteriza por el predominio de 
las mujeres, con una edad media de 72 años, casada, de etnia blanca declarada, con 5-10 años de educación, jubilada, con un ingreso promedio mensual de un salario mínimo.

Conclusiones. A través del instrumento de recolección se observó que la mayoría de las categorías de CIF seleccionadas se vio comprometida tanto con ligera dificultad o ninguna dificultad.

\section{RESUMO}

Os princípios do envelhecimento com qualidade de vida descrevem o idoso como pró-ativo, definindo seus objetivos e lutando para alcançá-los, reunindo recursos que são úteis na adaptação à mudança e ativamente envolvidos na manutenção do bem-estar.

O objetivo desta pesquisa é a caracterização do perfil dos idosos que participam do Centro de Convivência do Idoso ( $\mathrm{CCl}$ ) quanto ao sexo, idade, estado civil, etnia autodeclarada, escolaridade, ocupação, renda individual, número de filhos, naturalidade, condições de moradia, meio de locomoção que utiliza para ir ao $\mathrm{CCl}$ e atividade física preferida e descrever as condições de saúde e desempenho nas atividades de vida diária dos idosos participantes de $\mathrm{CCl}$, segundo a Classificação Internacional de Funcionalidade, Incapacidade e Saúde(CIF).

Método: Pesquisa do tipo exploratória, descritiva, transversal, com abordagem quantitativa, realizado em CCls do Vale Paraíba, São Paulo, Brasil. Foram incluídos no estudo aqueles que atendiam aos critérios de inclusão com idade igual ou superior a 60 anos, participantes, há pelo menos três meses completos e no máximo um ano dos CCls, que constituíram o campo de pesquisa. $\mathrm{O}$ estudo caracterizou-se pelo predomínio de mulheres, idade média de 72 anos, casadas, etnia autodeclarada branca, com 5-10 anos de educação escolar, aposentadas, com média de renda mensal de um salário mínimo.

Conclusôes. Por meio do instrumento de coleta foi possível observar que a maior parte das categorias da CIF selecionadas era pouco comprometida com dificuldade leve ou sem dificuldade.

\section{ABSTRACT}

The principles of aging and quality of life describe the elderly as proactive, setting goals and striving to achieve them, gathering resources that are useful in adapting to change and actively involved in maintaining wellness.

The goal of this research is to characterize the profile of the elderly participating in the Elderly Community Centre regarding gender, age, marital status, self-reported ethnicity, education, occupation, individual income, number of children, nationality, living conditions, means of transportation you use to go to the Elderly Community Centre and preferred physical activity and describe the health and performance in activities of daily living of the elderly participants from JRC, according to the International Classification of Functioning, Disability and health (CIF).

Method: The research was exploratory, descriptive, cross-sectional quantitative approach carried out in Elderly Community Centre Paraíba Valley, São Paulo, Brazil. The study included those who met the inclusion criteria of age or over 60 years, participating for at least three months and complete within one year of the Elderly Community Centre, which were the search field. The study was characterized by the predominance of women, mean age 72 years, married, self-reported ethnicity white, with 5-10 years of education, retired, with an average monthly income of a minimum wage.

Conclusions: Through the collection instrument was observed that most of Elderly Community Centre categories selected was somewhat compromised with slight difficulty or no difficulty.

\section{INTRODUCCIÓN}

Siguiendo los patrones observados en otros países ${ }^{(1)}$, en Brasil el descenso de la fecundidad y el aumento de la esperanza de vida dieron como resultado en los últimos 
30 años, el aumento significativo en la población anciana. Además, la mejora relativa en el acceso de la población a los servicios de salud, las campañas nacionales de vacunación, elevando el nivel de educación de la población, las inversiones en infraestructuras de saneamiento y la percepción de los individuos en relación a la enfermedad son un conjunto de factores que contribuyen a aumentar la esperanza de vida de las personas de edad. Del mismo modo, la alta fecundidad observada en el siglo XX, en los años 50 y 60 , y la caída de la mortalidad que benefició a todos los grupos de la población llevó a la delimitación de este perfil demográfico ${ }^{(2-3)}$.

Es de destacar que el proceso de envejecimiento humano no es homogéneo y está influenciada por un número de aspectos, por ejemplo, género, etnia y las condiciones socioeconómicas de la población ${ }^{(4)}$.

Así, el tema envejecimiento comenzó a ganar espacios en otros campos del conocimiento.

Los principios de un envejecimiento con calidad de vida describen al anciano como proactivo mediante el establecimiento de metas y luchando por alcanzarlas, la recolección de recursos que son útiles en la adaptación a los cambios y participar activamente en mantener el bienestar. Este modelo comprende desde la satisfacción con la vida a los modelos basados en los conceptos de independencia, control, habilidades sociales y cognitivas ${ }^{(5)}$.

El sedentarismo en el anciano, a menudo, se debe más a las limitaciones socioculturales que a incapacidad funcional ${ }^{(6)}$. Las conductas atribuidas a las personas mayores se refieren a la pasividad e inmovilidad, con bajo nivel de actividad física. Sin embargo, se sabe que muchos de los resultados fisiológicos y funcionales observados en los ancianos son resultado de la inexistencia de estímulos más que de alteraciones atribuidas al envejecimiento ${ }^{(7)}$. Los estudios han demostrado los aportes positivos de los programas de actividad física para mejorar la salud general física y funcional de las personas mayores ${ }^{(8)}$.

Por lo tanto, un estilo de vida activo mejora la salud física y mental y contribuye al control de trastornos tales como depresión y demencia. Existe evidencia de que los ancianos físicamente activos tienen una menor prevalencia de las enfermedades mentales que los no-activos ${ }^{(9)}$.

La actividad física debe ser de fácil realización y no causar daños. Debe ser de bajo impacto y de intensidad moderada lo que significa la percepción del esfuerzo percibido, aumento del ritmo cardíaco y/o la frecuencia respiratoria, permitiendo que el individuo respire sin dificultad y con aumento de la temperatura del cuerpo ${ }^{(8)}$.

Los grupos de convivencia ${ }^{(10)}$ proporcionan medios de dar poder a los ciudadanos en su cotidiano a través de una reflexión de su entorno sociocultural suscitando mecanismos individuales y colectivos de intervención en la vejez. Estos grupos fueron diseñados con objetivos, actividades y propuestas diferenciados, con espacios para el ocio, la sociabilidad, la cultura y para la construcción de una conciencia cívica.

Estos grupos realizan diversas actividades de cuño recreativo, cultural, social, educativo y de salud, orientadas por profesionales expertos que participan en la promoción de la salud y el envejecimiento activo. 
Con el objetivo de satisfacer las necesidades de saber más acerca de las consecuencias de la enfermedad y el impacto en la vida cotidiana de las personas, en 1976, la Organización Mundial de la Salud (OMS) publicó la Clasificación Internacional de Deficiencias, Discapacidades y Minusvalías (CIDDM), de forma experimental. Esto se tradujo al portugués como Clasificación Internacional de Deficiencias, Discapacidades y Minusvalías (handicaps) (CIDID) ${ }^{(11)}$.

De acuerdo con este marco conceptual, el deterioro (discapacidad) se describe como anomalías en los órganos y sistemas y estructuras del cuerpo; la discapacidad se caracteriza por las consecuencias de un fracaso en términos de rendimiento funcional, - la realización de actividades; handicap (desventaja) refleja la adaptación del individuo al medio ambiente como resultado de la deficiencia y la incapacidad ${ }^{(12)}$.

Claramente $^{(13)}$, se conceptualizó la Clasificación Internacional del Funcionamiento de la Discapacidad y la Salud (CIF) como una clasificación que describe el funcionamiento y la discapacidad relacionada con las condiciones de salud, la identificación de lo que una persona "puede o no puede hacer en su vida diaria" teniendo en cuenta las funciones de los órganos o sistemas y estructuras del cuerpo, así como las limitaciones de las actividades y la participación social en el entorno en el que vive la persona.

La CIF describe la situación de cada persona dentro de una gama de áreas de salud o relacionadas con la salud, que nos ofrece un lenguaje común y un modelo conceptual internacional universal para describir la salud y la discapacidad ${ }^{(14)}$.

No es una clasificación de las personas, ya que permite describir las características de la persona en las diferentes áreas y las características de su entorno físico y social, la selección de un conjunto de códigos que documenta la funcionalidad de su perfil y la participación. Hay varios países que han adoptado el marco CIF en los cambios legislativos y de regulación política y social en el contexto de las pensiones de invalidez, pensiones, políticas o reformas laborales de personas con discapacidad $^{(15)}$.

La CIF pone todas las enfermedades y problemas de salud en condiciones de igualdad, independientemente de sus causas. Una persona no puede ir a trabajar debido a un resfriado o a la angina de pecho, pero también a causa de una depresión. Este enfoque neutro coloca los trastornos mentales en el mismo nivel de la enfermedad física y ayuda a reconocer y establecer la carga mundial de morbilidad asociada con problemas depresivos, que actualmente representan la primera causa de años de vida perdidos por discapacidad ${ }^{(15)}$.

\section{OBJETIVOS}

Analizar el estado y el rendimiento en actividades de la vida diaria de los participantes de mayor edad del Centro Comunitario de Personas Mayores de la Clasificación Internacional del Funcionamiento, la Discapacidad y la Salud ${ }^{(15)}$;

Caracterizar el perfil de las personas mayores que participan en el Centro Comunitario de Personas Mayores con respecto a sexo, edad, estado civil, origen étnico auto-reporte, educación, ocupación, ingresos personales, número de hijos, la nacionalidad, la vivienda, los medios de locomoción que utiliza para ir al Centro Comunitario de Personas Mayores y la actividad física preferida. 


\section{MÉTODO}

La investigación fue de tipo exploratorio, descriptivo, transversal, enfoque cuantitativo, realizado en los Centros de Mayores de Paraíba Valley, Estado de São Paulo, sureste de Brasil. Las siete ciudades se eligieron por conveniencia del investigador.

En el muestreo por conveniencia, esta muestra se compone de miembros de la población que tienen mayor disponibilidad para la investigación o con las que el investigador tiene fácil acceso ${ }^{(16)}$.

El Valle del Paraíba es una región socioeconómica del estado oriental de São Paulo y el oeste del estado de Río de Janeiro, lo que significa concentrar una porción considerable del Producto Interno Bruto (PIB) de Brasil. El nombre se debe al hecho de que la región es la parte inicial de la cuenca del río Paraíba do Sul, hay que destacar que el nombre se refiere solamente a una región con determinadas características socioeconómicas, ya que el río Paraíba do Sul se extiende a lo largo de casi toda la extensión del estado de Río de Janeiro y separa parte del estado de Minas Gerais. Su ubicación se encuentra a orillas de la Carretera Presidente Dutra (BR-116), exactamente entre las ciudades de Río de Janeiro y São Paulo, dentro de la megalópolis formada por las dos capitales. La población agregada de todas las ciudades de la región es de casi 3,3 millones de personas ${ }^{(3)}$.

La población total de personas mayores de las siete ciudades es de 40.928. Se establecieron criterios de inclusión y exclusión para la selección de las personas mayores que podrían participar en el estudio, lo que se tradujo en 2.339 individuos. Para la recolección de datos y el cálculo del tamaño de muestra se realizó con el significado computacional de un $5 \%$, lo que resulta en el número de 386 personas mayores que, por sorteo, formaron la muestra de esta investigación.

Este proyecto fue presentado a la Comisión de Ética (CEP) de las Facultades Integradas Teresa D'Avila (Fatea) aprobado bajo n.87/2009.

Los criterios de exclusión fueron relacionados con los participantes de mayor edad con menos de tres meses completos y más de un año del Centro Comunitario de Personas Mayores y que se niegan a participar como voluntarios, firmando Término de Consentimiento. Criterios definidos para la adaptación de las personas mayores al Centro Comunitario de Personas Mayores.

Los siguientes instrumentos, que se describen a continuación, se utilizaron para recolectar datos: La lista de CIF y Mini Examen del Estado Mental.

Los datos fueron analizados estadísticamente de modo descriptivo, con un enfoque en la funcionalidad y la discapacidad relacionada con las condiciones de salud, teniendo en cuenta las funciones de los órganos y estructuras del cuerpo y sistemas, así como las limitaciones de las actividades y la participación social en el entorno en el que la persona vive.

Para la comparación de variables categóricas entre los grupos se utilizó la prueba exacta de Fisher para verificar la asociación entre el elemento en cuestión y la variable explicativa (género, edad, educación, Mini Examen del Estado Mental, número de hijos, las actividades físicas y los medios de transporte que utiliza para ir a $\mathrm{CCl}$ ), para valores esperados por debajo de 0,05. 
El nivel de significación para las pruebas estadísticas fue de $5 \%(p<0,05)$.

\section{RESULTADOS Y DISCUSIÓN}

Esta es la primera descripción del funcionamiento y la discapacidad a través de las categorías de la CIF en los ancianos, en una muestra brasileña.

El estudio se caracteriza por el predominio de las mujeres, con una edad media de 72 años, casada, etnia blanca autodeclarada, con 5-10 años de educación, jubilada, con un ingreso promedio mensual de un salario mínimo, dos hijos, natural de la ciudad de recogida de datos, reside en casa propia, el medio de locomoción para ir al Centro Comunitario de Personas Mayores y la actividad física es caminar, con preservación de la función cognitiva.

En el estudio ${ }^{(17)}$ con 184 personas mayores en un centro comunitario en Minas Gerais hubo predominio del sexo femenino (88,6\%), faja de edad entre 60 y 69 años $(58,7 \%)$, en la escolaridad predominó el primer grado incompleto $(56,5 \%)$ en el arraigo domiciliario $(78,8 \%)$ informaron de que vivían juntos y en la situación laboral, la mayoría $(46,7 \%)$ pertenece al grupo de jubilados y pensionistas; corroborando el estudio en cuestión.

Acerca de los niveles de condición física de los adultos ancianos ${ }^{(18)}$ de ambos sexos se encontró que las mujeres mayores presentaron resultados significativamente más altos que los de los hombres. Por lo tanto, la posibilidad de mejoras en las mujeres con la práctica regular de actividad física es baja ya que presentan valores iniciales de flexibilidad en comparación con los varones

En este estudio, las categorías de la CIF de las funciones de la energía y el impulso, moverse con algún tipo de equipamiento, dirigir vehículos de motor y educación escolar, poseen significación para ambos sexos (masculino y femenino).

El hecho de tener una mayor presencia de los ancianos entre 60 y 69 años en los grupos de convivencia se asocia con una menor autonomía y participación en el mercado de trabajo ${ }^{(19)}$, con tiempo libre para actividades de ocio, o con un escape del estereotipo de las personas mayores. Por otra parte, la baja participación de las personas mayores de 80 años puede deberse al mayor grado de comorbilidad con el aumento de la edad y la dependencia. Estos factores son importantes porque pueden limitar el acceso y la participación en las actividades que ofrece el proyecto, ya que estas personas mayores dependen de otros para llevarlos al lugar de las reuniones.

En este grupo de edad, encontramos ancianos fuera del mercado de trabajo, así que con más tiempo libre para realizar actividades dentro del Centro Comunitario de Personas Mayores ${ }^{(19)}$, los viejos estereotipos de la vejez ya no son aprobadas por las personas mayores. Ya no aceptan "quedarse en casa cuidando de los nietos" o haciendo ganchillo, buscan nuevas actividades que traigan bienestar.

Respecto a la edad, hubo significación estadística para las siguientes categorías de CIF - Funciones de la energía y el movimiento, las funciones emocionales, escuchar, habilidad con las manos, agarrar, sostener, relaciones familiares y solución de problemas. 
Un estudio realizado en la India ${ }^{(20)}$ mostró un total del $85 \%$ de una población de 356 ancianos encuestados con una edad media de 75 años, problemas médicos, deterioro cognitivo y sensorial, $27 \%$ con discapacidad leve en cualquier campo y el $31 \%$ restante tenía un mayor nivel de discapacidad.

Hay una mayor incidencia de caídas en mujeres menores de 75 años, después de esta edad la probabilidad es similar en ambos sexos ${ }^{(21-22)}$. Este hecho sigue siendo poco conocido en la literatura, aunque se ha sugerido como causas de las caídas la mayor fragilidad de las mujeres en relación con los hombres, así como una mayor prevalencia de enfermedades crónicas y la mayor exposición a las actividades domésticas, aumentando así la posibilidad de caídas.

Para los ancianos con diferentes años de escolaridad hubo significancia para las funciones de energía e impulso, levantar y cargar objetos, adqusición de bienes y servicios, interacciones interpersonales, relaciones sexuales, operaciones básicas de la vida económica y Vida política y ciudadanía.

De los 230 adultos mayores estudiados del Centro de Convivencia de la ciudad de Campina Grande-PB, $184(80,0 \%)$ eran mujeres y 46 (20,0\%) hombres. Mostró las siguientes características: edad entre 60 y 87 años, con mayor frecuencia en el grupo de edad de 71 a 80 años, 88 (38,4\%), siendo más frecuente viuda $89(39,2 \%)$, nivel de educación primaria incompleta fue el más común 110 (48,0\%), el analfabetismo sigue apareciendo en $60(26,2 \%)$ de las personas mayores. En cuanto a los ingresos mensuales individuales se distribuyen en bandas de un salario mínimo $23(10,0 \%)$, uno a tres salarios mínimos $180(78,6 \%)$, más de tres salarios $6(2,6 \%)$ y sin ingresos $70(8,7 \%)^{(23)}$. El salario mínimo en Brasil es de 236.62 EUR.

Un estudio con 1216 ancianos rurales en el noreste de España mostró que el 85\% de los participantes eran casados o viudos, que viven en sus propias casas con sus familias y eran de baja o media baja clase social. El nivel educativo de la muestra del estudio fue bajo, con un promedio de 7,8 años de escolaridad ${ }^{(24)}$.

En este estudio presentaron significancia para ancianos con diferente número de hijos las categorías movimientos involuntarios, conversación, cuidado de los objetos del hogar, las relaciones con los extranjeros, profesionales de la religión, salud y actitudes individuales de los profesionales de la salud.

En una encuesta realizada en la ciudad de São Paulo ${ }^{(25)}$, se dio una característica similar a la encontrada en este estudio, en el que la mayoría de la gente vive en hogares con hijos y nietos, y este tipo de arreglo llamado multigeneracional parece ser una característica de los países en desarrollo como Brasil.

El crecimiento de la proporción de personas que viven solas $(8,3 \%$ a $11,1 \%)$ en la Encuesta Nacional por Muestra de Domicilios es una tendencia que se ha visto en los últimos años y se considera el resultado de la reducción en las tasas de mortalidad y esperanza de vida, especialmente para las mujeres. En 2007, los arreglos familiares proprietorships representaron alrededor de 6,7 millones de dólares, de los cuales $(40,8 \%)$ se componía de personas de 60 años o mayores. La situación laboral, respectivamente, en la región sudeste es de aproximadamente $(40,9 \%)$ de los ingresos de los hogares de la jubilación de las personas de edad ${ }^{(4)}$. 
En cuanto a la actividad física de elección para las personas mayores que participan en los Centros Comunitarios de Personas Mayores del Valle del Paraíba - SP se asoció con el valor de p (prueba exacta de Fisher) para las funciones Categorías de la CIF funciones de visión, funciones urinarias, calcular, levantar y llevar objetos, las relaciones familiares, relaciones íntimas y personas con posiciones de autoridad.

En un Centro Comunitario de Personas Mayores de una ciudad de Paraíba Valley, en Sao Paulo, con 44 independientes de edad avanzada, media de 70 años (33\%) y casados $(84,09 \%)$ informaron ejercicio RAM físico dos veces a la semana ${ }^{(26)}$.

Los resultados están en línea con un estudio ${ }^{(27)}$, cuando se refiere precisamente a que el caminar, la gimnasia y la natación son las principales actividades físicas de las mujeres mayores. Además, caminar y hacer ejercicio son las formas más populares de actividad en las personas mayores de Finlandia, donde 2/3 de la población camina regularmente y $1 / 3$ hace gimnasia en casa, a pesar de que la participación en estas actividades comienza a disminuir en las personas mayores de 80 años o más.

Nos dimos cuenta de que la mayoría de las categorías de CIF estudiadas en esta muestra reportó poco deterioro de la función con dificultad leve o sin dificultad, lo que se espera para personas mayores independientes (100\%) que participan en actividades sociales normales $(100 \%)$, que se caracteriza por ser mujeres $(80 \%)$, casadas $(60 \%)$ con una edad media de 72 años.

El polémico debate sobre las diferencias entre hombres y mujeres aumenta cuando se observa que en los países donde las mujeres sufren un mayor dominio del hombre es donde se dan las mayores diferencias en la esperanza de vida por sexo, contrariamente a lo que ocurre en los países donde la mayor independencia de la mujer está acompañada por diferencias mayores. Esto sucede probablemente porque estas mujeres han adquirido mayor conocimiento de las medidas preventivas de salud (nutrición adecuada y ejercicio) y también proporciona una mayor independencia que las mujeres buscan atención médica adecuada ${ }^{(28)}$.

Un estudio realizado por la Fundación Oswaldo Cruz, de 1990 a 2002, en que fueron identificados en 35 programas de promoción de la salud para los ancianos, se accesaron en realidad 20 estudios (11 internacionales y nueve brasileños). Un estudio cuasi-experimental predomina en la experiencia internacional, mientras que en Brasil son comunes los relatos de experiencia. En los programas internacionales, la mayoría de la población anciana en cuestión está relativamente bien, en el sentido de tener un seguro de salud, tienen alto nivel educativo y el estado de salud y los ingresos por encima de la media tres. La mayoría de los experimentos en Brasil se desarrolla en los servicios de salud pública, vinculada a la asistencia regular, y los ancianos tienen bajo nivel educativo con altas tasas de analfabetismo. En general, los objetivos de los programas convergen en el horizonte para mejorar la salud y calidad de vida de las personas mayores, con énfasis en las dimensiones de los programas de autocuidado, talleres o conferencias y grupos de marcha. Sin embargo, los subgrupos, como los ancianos residentes de hogares de ancianos, rurales y de grupos étnicos fueron excluidos de la evaluación de los programas de promoción de la salud ${ }^{(29)}$.

Las funciones corporales con más deficiencias son las funciones de sueño (32\%) y las funciones emocionales (40\%) en consonancia con otros resultados reportados en la literatura en los ancianos de baja escolaridad y funciones cognitivas poco desarrolladas. Por otra parte, las funciones del cuerpo relacionadas con la movilidad 
de las articulaciones y la fuerza muscular (40\%), las funciones relacionadas con el tono muscular (46\%), las funciones relacionadas con los movimientos involuntarios (62\%) fueron las que habían conservado más completa frecuencia (sin discapacidad), lo que puede indicar resultado satisfactorio debido al desarrollo y a la participación de las personas de edad en actividades físicas propuestas por el Centro Comunitario de Personas Mayores con el propósito de un envejecimiento activo y saludable.

Los recursos personales y sociales de que el individuo dispone es una manera de determinar las consecuencias de la discapacidad funcional, por tanto, es importante evaluar no sólo el deterioro funcional, sino cómo tales limitaciones son experimentadas por cada uno ${ }^{(30)}$.

En este estudio, los resultados del Examen de Estado Mini-Mental se asociaron significativamente a las funciones de la energía y el pulso, las funciones de atención, funciones de memoria, las funciones emocionales, funciones de visión, sensación de dolor, las funciones urinarias, observar, realizar múltiples tareas, comunicación recepción de mensajes orales, comunicación recepción de mensajes no verbales, habla, producción de mensajes no verbales, levantar y transportar objetos, manos hábiles, Andar, desplazarse usando algún tipo de equipamiento, conducir, conducción de transporte de tracción animal, conducción de vehículos motorizados, Lavado, Cuidado de partes del cuerpo, los procesos de atención relacionados con la excreción, vestirse, beber, cuidar de la propia salud interacciones interpersonales complejas, relaciones con desconocidos, relaciones formales, relaciones amorosas, relaciones maritales, La educación informal, el trabajo, la movilidad y el transporte personal en espacios interiores y exteriores, y productos de tecnología para las actividades culturales, recreativas y deportivas, productos y tecnología para la práctica religiosa y la vida espiritual, Familia inmediata, amigos, conocidos, compañeros, colegas, vecinos y miembros de la comunidad, las personas de autoridad, asistentes personales y cuidadores, profesionales de la salud, otros profesionales, las actitudes individuales de los profesionales de la salud, las actitudes individuales de los profesionales relacionados con la salud, servicios, sistemas y políticas de seguridad social, servicios, sistemas y políticas de salud.

Las relaciones familiares: crear y mantener relaciones de parentesco, con miembros de la familia, parientes, familia adoptiva y de creación y parientes no consanguíneos, relaciones más distantes como primos segundos o tutores legales (D760). Incluye: las relaciones entre padres e hijos e hijos y padres, entre hermanos y otros miembros de la familia.

Las relaciones íntimas: la creación y el mantenimiento de las relaciones románticas o íntimas entre las personas, como esposos, amantes o parejas sexuales (D770). Incluye: relaciones sentimentales, conyugales y sexuales.

Las relaciones románticas: la creación y el mantenimiento de una relación basada en la atracción física y emocional, que puede conducir a relaciones íntimas a largo plazo (d7700).

Las relaciones maritales: la creación y el mantenimiento de una relación íntima de carácter jurídico con otra persona, como en el matrimonio legal, además de ser o convertirse en una esposa o esposo cónyuge legalmente casado o una mujer soltera (d7701). 
Sexo: crear y mantener una relación sexual con su cónyuge o compañero (d7702).

En los países occidentales, aproximadamente el 30\% de las personas mayores de 65 años se caen al menos una vez al año, y aproximadamente la mitad sufre dos o más caídas. Esta frecuencia es menor en los países del Este, donde aproximadamente el $15 \%$ de la caída de ancianos ocurre una vez al año y sólo el 7,2\% es caída recurrente. Este evento es la sexta causa principal de muerte entre las personas mayores de 65 años, y los resultados incluyen lesión no mortal, el miedo a caer otra vez, la discapacidad funcional y la institucionalización. En Brasil, un estudio realizado en São Paulo mostró que la frecuencia de las caídas es del 32,7\% y las caídas recurrentes en la comunidad de edad avanzada fue del $13,9 \%{ }^{(22)}$.

También en cuanto al cuidado personal ${ }^{(31)}$, comer y beber son tareas de menor complejidad. Así, los ancianos obtienen un mejor nivel de desempeño en estas tareas.

En cuanto a las actividades e intereses, se observa que las categorías resolver problemas, habla y trabajo remunerado (76\%), producción de mensajes no verbales (82\%), adquisición de una vivienda y compra de bienes y servicios (90\%); caminar, moverse con algún tipo de equipo, lavado, cuidado de partes del cuerpo, los procesos de atención relacionados con la excreción, vestirse, el cuidado de su propia salud, preparación de comidas, limpieza, cuidado de los objetos hogar, ayudar a otros, las relaciones formales, educación informal, y la religión, transacciones económicas básicas y religión y espiritualidad (98\%), comer, beber, interacciones interpersonales básicas, relación con los extranjeros, las relaciones sociales informales, vida comunitaria, recreación y tiempo libre, Derechos Humanos y vida política y ciudadanía $(100 \%)$ fueron los menos frecuentemente comprometidos, y tales actividades se realizan con mayor frecuencia lo que proporciona más habilidad, ya que las categorías Hacer múltiples tareas (32\%), la comunicación-recepción de mensajes orales $(30 \%)$, levantamiento y transporte de objetos (32\%) y Autosuficiencia (36\%) tuvieron la mayor limitación o restricción.

El proyecto Salud, Bienestar y Envejecimiento (SABE), que es un estudio multicéntrico realizado en siete ciudades de América Latina y el Caribe con personas de 60 años o más, investigó cuestiones importantes acerca de la funcionalidad y de las actividades básicas e instrumentales de la vida diaria. La prevalencia de la discapacidad para realizar al menos una actividad básica de la vida diaria (AVD), medida desde el informe de la presencia de dificultad fue de $18,6 \%$ en Ottawa, el $13,8 \%$ en Bridgetown, el $23,7 \%$ en São Paulo, el 22,3\% en Toronto, el $20,5 \%$ en La Habana, el $19,4 \%$ en México y $16,8 \%$ en Montevideo. Para las actividades instrumentales de la vida diaria (AIVD), las prevalencias fueron $28,7 \%, 23,5 \%, 40,3 \%$, $31,8 \%, 27,8 \%, 28,6 \%$ y $17,0 \%$ respectivamente. Las variables asociadas a la incapacidad funcional fueron: mayor número de enfermedades crónicas, artrosis, enfermedades cardiovasculares y depresión, ser mujer, tener edad avanzada, baja auto-evaluación del estado de salud y deterioro cognitivo. Las asociaciones más fuertes se encuentran en relación con las actividades instrumentales ${ }^{(32)}$.

Estudio $^{(33)}$ realizado en el Reino Unido verificó una orden de restricción de actividades, comenzando con el cuarto de baño, caminar, higiene vestirse y alimentación. El estudio longitudinal ${ }^{(34)}$ realizado en los Estados Unidos con 5.151 personas mayores mostró una disminución de la capacidad funcional, especialmente en relación con baño, higiene, cuidado personal, vestirse y movilidad. 
En Brasil, hay estudios que analizan de forma aislada cada grupo de actividades de la vida diaria. En general, la investigación evalúa las tareas en forma de bloque, un hecho que dificulta profundizar en la cuestión. Se sabe que los residentes ancianos de la comunidad son más activos y por lo tanto tienen un nivel funcional por encima de los que viven en residencias geriátricas. Entender el comportamiento funcional dentro de cada grupo puede facilitar la comprensión de los temas involucrados en el deterioro funcional y servir como fuente de información para acciones específicas para cada situación, ya que el tipo de ayuda que necesita, las demandas de servicios y los costes de la discapacidad son muy diferentes ${ }^{(35)}$.

Para los ancianos de este estudio que usan un medio de transporte para ir al Centro Comunitario de Personas Mayores, hubo significancia de las Funciones de visión, adquisición de bienes, Calcular, Levantar y llevar objetos, relaciones familiares, relaciones íntimas, y las personas con una posición de autoridad tiene importancia, porque promueven su acceso al transporte.

Sin embargo, esta política ha contribuido poco a la construcción de la ciudadanía, pues aquellos que están por debajo del umbral de la pobreza tienen tantas necesidades básicas insatisfechas que un salario mínimo no es suficiente para garantizar una vida digna. Estudios ${ }^{(36)}$, entre otros, demuestran la insuficiencia de nuestro salario mínimo, que sólo contempla una cesta, el establecimiento de la línea de indigencia y la reducción de las necesidades de alimentación humana.

Categorías de los diferentes capítulos del componenete Actividades e Intereses se han descrito como teniendo algún grado de dificultad, desde actividades de aprendizaje hasta el habla, la movilidad y otros. Esto indica la necesidad de equipos multidisciplinarios en los programas de atención de salud para los ancianos y gerontología.

Los factores ambientales en el CIF fueron descritos como constitutivos de un entorno físico y las actitudes sociales en las que las personas viven y conducen sus vidas. La codificación de estos factores contextuales debe ser considerada para cada componente de la operación y codificar de acuerdo con estos componentes. Una lista de Factores Ambientales es el primer componente de los factores contextuales. Los factores ambientales tienen un impacto en todos los componentes de funcionamiento y la discapacidad y están dispuestas en secuencia, el entorno inmediato del individuo con el medio ambiente general ${ }^{(15)}$.

En este estudio la mayoría de las categorías de factores ambientales ha sido calificada como facilitadora, lo que indica que estas mujeres no se dan cuenta de muchas barreras ambientales en sus actividades diarias. Esto puede deberse al hecho de que en su mayoría son saludables y son capaces de utilizar los bienes y servicios que se crean a partir de un modelo tradicional de diseño y planificación que considera a las personas sin discapacidad como parámetros. Se puede proponer que si la muestra estaba compuesta por personas con discapacidad de edad avanzada, 0 menos activa, un mayor porcentaje de categorías de factores ambientales serían identificados como barrera.

En este estudio no hubo calificación 8 (sin especificar) en los 386 ancianos que respondieron a 107 categorías de CIF $(107$ x $386=41.302)$. 
En un estudio realizado en Brasil ${ }^{(37)}$ con mujeres con fibromialgia, observaron veintinueve participantes en una encuesta, y de todas las respuestas posibles (67 categorías x 29 pacientes $=1943$ ), sólo 14 obtuvieron la calificación de 8 (sin especificar), lo que indica una buena calidad de cumplimentación de la misma, pues los core sets se rellenaron casi por completo.

\section{CONCLUSIONES}

El estudio se caracteriza por el predominio de las mujeres con una edad media de 72 años, casada, de etnia blanca autodeclarada, con 5-10 años de educación, se retiró con un ingreso medio mensual de un salario mínimo, dos hijos, natural de la ciudad de recolección de datos, reside en casa propia, medio de locomoción para ir al $\mathrm{CCl}$ y actividad física preferidos es caminar, con función cognitiva conservada, sin cambios en la presión arterial y la respiración.

A través de instrumentos de recolección de información se observó que la mayoría de las categorías de CIF seleccionadas se vio comprometida tanto con ligera dificultad, y sin dificultad, lo que se espera en una muestra independiente de personas mayores $(100 \%)$ que participan en las actividades sociales habituales (100\%), que se caracteriza por mujeres (80\%), casadas $(60 \%)$ con una edad media de 72 años, como se ha señalado.

Las funciones corporales con más deficiencias son las las funciones de sueño (32\%) y las funciones emocionales (40\%) en consonancia con otros resultados reportados en la literatura en los ancianos de baja escolaridad y funciones cognitivas poco desarrolladas. Además, las funciones del cuerpo de las Funciones relacionadas con la movilidad de las articulaciones y la fuerza muscular (40\%), las funciones relacionadas con el tono muscular (46\%), las relacionadas con los movimientos involuntarios (62\%) fueron las que habían conservado completamente preservadas con más frecuencia (sin discapacidad), lo que puede indicar el resultado satisfactorio que resulta de las actividades físicas desarrolladas en el Centro Comunitario de Personas Mayores.

La aplicación de la lista de comprobación del CIF en ancianos independientes en los CIFs mostró una población practicante de actividades sociales regulares, sin discapacidad, es decir, completamente preservadas las funciones de la conciencia, la orientación y las intelectuales, pero afectadas las funciones del sueño, emocional y cognitivo superior. Para las actividades y participaciones, resolver problemas, hablar, caminar, desplazarse utilizando algún tipo de equipamiento, la atención de las partes del cuerpo, ayudar a otros, la educación informal y el trabajo remunerado, se vieron afectados con menor frecuencia, mientras que las tareas múltiples, comunicaciónrecepción de mensajes orales, levantar y transportar objetos y la autosuficiencia económica tuvo la mayor limitación o restricción.

En cuanto a las actividades e intereses, se observa que las categorías de problemas, habla y trabajo remunerado (76\%), los mensajes no verbales de producción (82\%), la adquisición de una vivienda y la compra de bienes y servicios (90\%), Caminar, moverse con algún tipo de equipo, lavado, cuidado de partes del cuerpo, los procesos de atención relacionados con la excreción, vestirse, el cuidado de su propia salud, preparación de comidas, hacer la limpieza, realización de las tareas domésticas, cuidado de los objetos del hogar, ayudar a otros, las relaciones formales, educación informal, transacciones económicas básicas y y la religión y espiritualidad (98\%), comer, beber, las interacciones interpersonales básicas, relación con extraños, 
relaciones sociales informales, vida comunitaria, recreación y tiempo libre, y Derechos Humanos y vida política y ciudadanía (100\%) fueron los menos frecuentemente comprometidas, siendo que tales actividades son realizadas con mayor frecuencia proporcionando más habiliidad, ya que las categorías Aprender a leer (30\%), Aprender a calcular (32\%), realizar tareas múltiples (32\%), la comunicación- recepción de mensajes orales (30\%), elevación y transporte de objetos (32\%) y la autosuficiencia (36\%) tuvieron la mayor limitación o restricción.

En este estudio no hubo calificación 8 (sin especificar) en 387 ancianos que respondieron 107 categorías de CIF $(107$ x $386=41.302)$.

\section{CONSIDERACIONES FINALES}

El éxito de los programas para las personas mayores que producen un discurso comprometido en revisar los estereotipos negativos de la vejez y abren espacios a las experiencias de envejecimiento exitoso vividas colectivamente, realizar proyectos abandonados en otras etapas de la vida, establecer relaciones más fructíferas con el mundo y al mismo tiempo, abre nuevos espacios para la articulación de las demandas políticas y establecer nuevos mercados de consumo.

Al ceñirnos a la cuestión de la especificidad y la singularidad de cada ser humano, asumimos la condición de que las políticas sanitarias y sociales deben ser propuestas para grupos de población, aunque, teniendo en cuenta las necesidades y experiencias de cada individuo.. Así, la edad se debe considerar en el ciclo de la vida no como una enfermedad sino como un proceso de vivr envejecinedo, de convivir con complicaciones, que pueden ser prevenibles y, en particular, pueden tratarse ${ }^{(38)}$.

Los perfiles de población cambiantes, en lo tocante a la franja de edad, ha hecho que los órganos oficiales modifiquen sus programas pasando a considerar a la tercera edad, una de las poblaciones objetivo de cualquier política oficial ${ }^{(38)}$.

Los programas de salud y sociales deben tratar de responder a la urgente necesidad de desmitificar los conceptos anteriores en cuanto a edad, basados en la ciencia del envejecimiento, para la construcción de las condiciones socioculturales que conducen a una vejez digna ${ }^{(38)}$.

Así, construiremos un país de ciudadanos, personas incluidas y acogidas en políticas sociales y de salud, sin importar su edad ${ }^{(38)}$.

El nuevo texto propuesto por la CIF es un paso importante hacia un cambio de paradigma en materia de discapacidad, haciendo hincapié en el importante papel de los factores contextuales, que no eran relevantes en los sistemas de clasificación anteriores. Incluso si sus codificaciones no son todavía de uso común en nuestro sistema de salud, CIF ya tiene el mérito de señalar una nueva dirección en la evaluación del impacto de la enfermedad sobre la vida de las personas y en el proceso de envejecimiento.

La CIF propone que la discapacidad funiconal, resulte no sólo de una deficiencia orgánica, sino de influencia mutua entre la disfunción presentada por el individuo como la limitación de sus actividades, restricción de participación social y como resultado de factores ambientales y personales que interfieren en el desempeño de las actividades de la vida diaria, y pueden actuar como barreras o facilitadores del 
estado funcional. Como resultado, las condiciones de vida extremadamente desfavorables determinan un mayor deterioro funcional y un peor rendimiento social. Los determinantes sociales de la salud también imponen barreras a la funcionalidad $^{(39)}$.

Los límites para la aplicación de la lista de comprobación CIF, según este estudio apuntan a la necesidad de capacitación para la implementación de la herramienta CIF para los profesionales y / o investigadores.

La aplicación de la lista de comprobación CIF no es ampliamente utilizada en las encuestas nacionales y en la implementación de políticas públicas en la gestión sanitaria. Esto también apunta ventajas tales como la comparación de los resultados con estudios internacionales, así como describe, compara y subvenciona las intervenciones de salud en las personas mayores en los países en desarrollo, especialmente para la formulación de políticas públicas que inserten a la tercera edad en el proceso de globalización con la mejor salud y la participación social.

Aunque la CIF puede ser considerada como un gran avance en el diseño y en cómo comunicarse con detalle los cambios que determinan un mayor o menor grado de deterioro de la salud de la persona, en la práctica se ha encontrado gran dificultad en el uso de esta forma de clasificación de las condiciones salud. Las evidencias científicas producidas en el país, desde su publicación, no han demostrado su aplicabilidad en la rutina diaria de los profesionales sanitarios. Los escasos estudios han sido idealizados para, como mucho, presentar datos de unos pocos casos ${ }^{(39)}$.

La producción nacional de los estudios que utilizan la CIF para estimar el deterioro funcional es muy limitada. En Scielo biblioteca virtual, entre los años 2005 a 2011, de los 13 artículos indexados por los términos discapacidad y funcionalidad son sólo siete artículos originales, que efectivamente utilizan la escala para clasificar los niveles de participación, funcionalidad y / o discapacidad, ya que la otros se dirigen a los aspectos teóricos de la aplicación de la escala conceptual. En el Pubmed base, también puede detectarse un gran número de artículos en conceptual teórico y superados en número por la aplicación de la escala para describir trastornos, limitaciones, restricciones o barreras y factores facilitadores ${ }^{(39)}$.

Hay pocos estudios nacionales con la aplicación del check list de la CIF con ancianos, por lo que este estudio es pionero en el estado de São Paulo y en Brasil.

El uso de una referencia, por muy compleja que sea su formulación, proporciona una comunicación rápida, directa y objetiva de una información muy útil para la planificación y gestión de recursos. Y la CIF proporciona también una comunicación rápida, directa y objetiva. ¿Por qué ante este escenario nacional y mundial de alta morbilidad por enfermedades crónicas no transmisimisbles que repercute en tasas elevadas de deficiencias, disfunciones y discapacidades, no promueve elevación similar de producción de datos utilizando esta escala? Después de diez años de su publicación CIF ha sido, de hecho, reconocida como una herramienta útil en la evaluación empírica de las alteraciones funcionales de los pacientes. Debemos reflexionar. Aunque muy bien elaborada, de hecho, su producción es operacionalmente poco ejecutable, exigiendo la evaluación por parte de diferentes profesionales para producir códigos precisos que implican varios constructos. Al igual que las lenguas no habladas, las escalas no usadas también mueren ${ }^{(39)}$. 


\section{REFERENCIAS}

1.Lloyd-Sherlock P. Population ageing in developed and developing regions: implications for health policy. Soc Sci Med; 2000, 51:887-95

2.Gordilho, A. et al. Desafios a serem enfrentados no terceiro milênio pelo setor saúde na atenção integral ao idoso. Rio de Janeiro: UnATI/UERJ; 2000.

3.IBGE - Instituto Brasileiro de Geografia e Estatística. Síntese de Indicadores Sociais Uma Análise das Condições de Vida da População Brasileira. Rio de Janeiro. 2007.

4. Brasil, Ministério da Saúde. Envelhecimento e saúde da pessoa idosa. Cadernos de Atenção Básica, Brasília, DF, n.19, 2006.

5. Inouye, K.; Pedrazzani, E.S.; Pavarini, S.C.I. Implicações da doença de Alzheimer na qualidade de vida do cuidador: um estudo comparativo. Cad. Saúde Publica vol.26 n.5. Rio de Janeiro. 2010.

6. Spirduso, W.W. Physical Dimensions of Aging. Human Kinetics Publishers, Champaign, Illinois, 1995.

7. Barry, H.C. e Eathorne, S.W. Exercise and aging. Issues for the practitioner. Medical Clinics North America, 1994, 357-375.

8. Mota, J.; Paiva, P.; Silva, S. Effects of a 6 month programme of nom specific physical activity on physical capacity of aged sedentary women. Journal of Human Movements Studies, 1995, 243-254.

9. Torres, A.C. et al. Ejercicios de resistencia: beneficios de la práctica sistemática en personas mayores. EFDeportes.com, Revista Digital. Buenos Aires, Año 15, № 148, Septiembre de 2010.

10. Costa, F.G; Campos, P.H.F. Práticas Institucionais e Representações da Exclusão na Terceira Idade. In Campos, P. H. F. ; Loureiro, M. C. S. (orgs.). Representações Sociais e Práticas Educativas. Goiânia-GO: EdUCG, pp. 187-207, 2003.

11. Secretariado Nacional de Reabilitação, Ministério do Emprego e da Segurança Social. Classificação Internacional das Deficiências, Incapacidades e Desvantagens (handicaps). Lisboa, 1989.

12. Buñuales, M.T.J.; Diego, P.G.; Moreno, J.M.M. La Clasificación Internacional del Funcionamento de la Discapacidad y de la Salud (CIF) 2001. Rev Esp Salud Publica, 76: 271-9, 2002.

13. Farias, N.; Buchalla, C.M. A Classificação Internacional de Funcionalidade, Incapacidade e Saúde da Organização Mundial da Saúde: conceitos, usos e perspectivas. Revista Brasileira de Epidemiologia, 8 (2), 187-193, 2005.

14. Organização Mundial da Saúde; Organização Panamericana de Saúde. Classificação Internacional de Funcionalidade, Incapacidade e Saúde. São Paulo: Editora da Universidade de São Paulo - EDUSP: 328p. 2003.

15. CIF: Classificação Internacional de Funcionalidade, Incapacidade e Saúde [Centro Colaborador da Organização Mundial da Saúde para a Família de Classificações Internacionais, org.; coordenação da tradução Cassia Maria Buchalla]. São Paulo: Editora da Universidade de São Paulo - EDUSP; 2003.

16.Brevidelli, M.M.; Domenico, E.B.L. Trabalho de Conclusão de Curso: Guia Prático para Docentes e Alunos da Área da Saúde. 1ª Ed. São Paulo: látria, cap. 2, 2006.

17. Hott, A.M.; Pires, V.A.T.N. Perfil dos idosos inseridos em um centro de convivência. Revista Enfermagem Integrada - Ipatinga: Unileste-MG - v.4 - n., jul./ago. 2011.

18, Botelho, R. Efeitos da pratica da atividade física sobre a Aptidão física de Adultos idosos. Dissertação apresentada às provas de Mestrado em Ciências do Desporto. Faculdade de Ciências do Desporto e de Educação Física da Universidade do Porto, 2002. 
19. Borges, P.L.C. et al. Perfil dos idosos frequentadores de grupos de convivência em Belo Horizonte, Minas Gerais, Brasil. Cad. Saúde Pública, Rio de Janeiro v.24, n.12, dez. 2010.

20.Srinivasan K., Vaz M., Thomas T. Prevalence of health related disability among community dwelling urban elderly from middle socioeconomic strata in Bangaluru, India. Indian J Med Res 131, April, 515-21, 2010.

21. Tinetti, M.E. Clinical practice: preventing falls in elderly persons. N Engl $\mathrm{J}$ Med. 348(1):42-9; 2003.

22. Perracini, M.; Ramos, L.R. Fatores associados a quedas em uma coorte de idosos residentes na comunidade. Rev Saúde Pública. 36(6):709-16; 2002.

23. Silva, A.D.L.; Catão, M.H.C.V. Doenças sistêmicas em idosos não institucionalizados. HU Revista, Juiz de Fora, v. 37, n. 3, p. 299-303, jul./set. 2012.

24. Pedro-Cuesta, J. et al. ICF-Based Disability Survey in a Rural Population of Adults and Older Adults Living in Cinco Villas, Northeastern Spain: Design, Methods and Population Characteristics. Neuroepidemiology. 35:72-82, 2010.

25. Ramos, L.R. Fatores determinantes do envelhecimento saudável em idosos residentes em centro urbano: Projeto Epidoso, São Paulo. Cad Saúde Pública. 19 (3): 793-8, 2003.

26. Ferreira, K.S. et al. Perception of the elderly toward sex in old age. Gereiatria \& Gerontologia. 3(4): 182-188, 2009.

27. Yusuf, $\mathrm{H}$. et al. Leisure-time physical activity among older adults. Arch. Internal Med. 156 (12)1321-1326; 2006.

28. Prata, L.E.; Yazaki, L.M. A dependência familiar do idoso nas populações de baixa renda. São Paulo: Fundação Seade, 1991.

29. Assis, M.; Hartz, Z.M.A.; Valla, V.V. Programas de promoção da saúde do idoso: uma revisão de literatura científica no período de 1990 a 2002. Ciência Saúde Coletiva. 9(3): 557-8, 2004.

30. Kjeken, I. et al. Activity limitations and participation restrictions in women with hand osteoarthritis: patients' descriptions and associations between dimensions of functioning. Ann Rheum Dis.64(11):1633-8; 2005.

31. Riberto, M. e cols. Reprodutibilidade da versão brasileira da Medida de Independência Funcional. Acta Fisiátrica, São Paulo, v. 8, n. 1, p. 45-52, 2001.

32. Menéndez, J. et al. In: Fragilidade no Idoso. Perracini MR, Fló CM. Fisioterapia: teoria e prática clínica. Funcionalidade e Envelhecimento. Editores da série: Celso R. F Carvalho e Clarice Tanaka, Guanabara Koogan, 2009.

33. Jagger, C. et al. Patterns of Onset of Disability in Activities of Daily Living with Age. American Geriatrics Society, New York, v.49, n. 4, p.404-409, 2001.

34. Dunlop, D.D.; Hughes, S.L.; Manheim, L.M. Disability in activities of daily living: patterns of change and a hierarchy of disability. American Journal of Public Health, Boston, v.87, n.3, p.378-383, mar., 1997.

35. Barbosa, A.R. et al. Functional limitations of Brazilian elderly by age and gender differences: data from SABE Survey. Cad Saúde Pública. 21: 177-85, 2005.

36. Sposati, A. "Pobreza e cidadania no Brasil contemporâneo". Revista Serviço social e Sociedade, n.63, p.131-139, 2000.

37. Riberto, M.; Saron, T.R.P.; Battistella, L.R. Resultados do core set da CIF de dor crônica generalizada em mulheres com fibromialgia no Brasil. Acta Fisiatr, 15(1): 6 12, 2008.

38. Fernandes, M.G.; Santos, S.R. Políticas Públicas e Direitos do Idoso: desafios da agenda social do Brasil contemporâneo. Achegas. net - Revista de Ciência Política n.34, mar/abr p. 49-60, 2007.

39. Fraga, M.H.; Sousa, C.S.; Ferreira, K.G.. Os debates ancestrais e atuais acerca do que é saúde e a classificação internacional de funcionalidade, incapacidade e 
saúde: contradições para compreensão das possibilidades de uma política nacional de saúde funcional. Revista Brasileira de Saúde Funcional, vol. 1. n.1 (1) p.1-11, 2011. 